\title{
Cardiac defibrillator implantation via persistent left superior vena cava - sometimes this approach is facile. A case report
}

Andrzej Bissinger, Fardokht Bahadori-Esfahani, Andrzej Lubiński

Department of Invasive Cardiology and Cardiodiabetology, University Hospital, Lodz, Poland

Submitted: 5 January 2010

Accepted: 27 February 2010

Arch Med Sci 2011; 7, 1: 161-163

DOI: 10.5114/aoms.2011.20623

Copyright @ 2011 Termedia \& Banach

\begin{abstract}
We report a case of persistent left superior vena cava (PLSVC) incidentally recognized during the implantation of a cardioverter-defibrillator. PLSVC is the most common venous anomaly of the thorax and drains into the right atrium. There are a lot of publications reporting success of pacemaker or defibrillator lead implantations via PLSVC. In this article we present the technique of approaching the right ventricle and right atrium via PLSVC; sometimes this method can be as straightforward as the classical way. Therefore, if PLSVC is recognized intra-operatively, we suggest continuing left-sided implantation, and considering a right venous access only in case of failure.
\end{abstract}

Key words: implantable cardiac-defibrillator, persistent left superior vena cava, defibrillator lead implantation.

\section{Introduction}

Persistent left superior vena cava (PLSVC) is a benign congenital vascular anomaly affecting $0.3-2 \%$ of healthy subjects [1]. The most common variation in this anomaly comprises $90 \%$ of all PLSVC reports presenting with both right and left superior vena cava. The PLSVC drains into the right atrium directly or most often through a dilated coronary sinus [1]. Isolated PLSVC is usually not recognized until the left cephalic or subclavian approach is used for diagnostic or therapeutic transcatheter procedures. Transvenous introduction of the lead from the right atrium to the right ventricle through the PLSVC may become a technically difficult procedure. It is often very time consuming and requires lengthy $\mathrm{X}$-ray exposure. This paper is a case report of successful cardiac-defibrillator (ICD) implantation via the PLSVC.

\section{Case report}

A 39-year-old male with cardiomyopathy and left ventricle ejection fraction (LVEF) $20 \%$ in functional status NYHA class II with a history of ventricular tachycardia and paroxysmal atrial fibrillation was referred to our department for cardioverter-defibrillator implantation. Before implantation, nuclear magnetic resonance (NMR) examination was performed. On the basis of NMR, the diagnosis of dilated cardiomyopathy

\author{
Corresponding author: \\ Andrzej Bissinger MD \\ Department of Invasive \\ Cardiology and \\ Cardiodiabetology \\ University Hospital \\ Zeromskiego 113 \\ 90-549 Lodz, Poland \\ Phone/Fax: +48 426393563 \\ E-mail: abissinger@gmail.com
}


after myocarditis was established. This examination showed severe left ventricular dilation $(80 / 69 \mathrm{~mm})$ and dysfunction - LVEF was $15-20 \%$ with global hypokinesis. Examination of blood flow to the heart was not performed because no congenital thoracicabdominal abnormalities were considered.

During ICD implantation, puncture of the left subclavian vein revealed PLSVC. Venography showed that this vein drains into the right atrium through a dilated coronary sinus (Figure 1). The typical ICD 1-coil active lead 6935 (Medtronic, Tolochenaz,

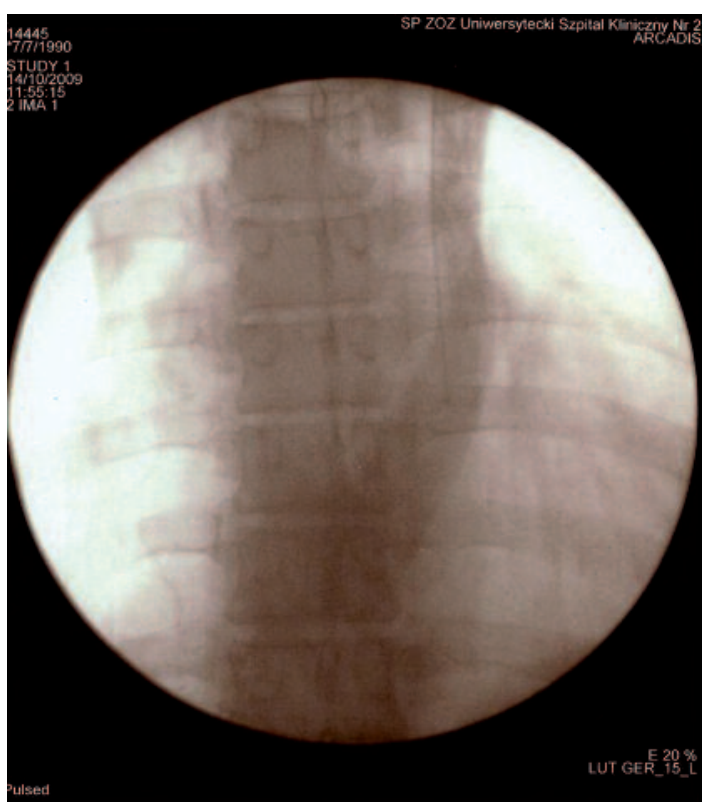

Figure 1. Venography demonstrating persistent left superior vena cava (PLSVC) drains into the right atrium through a dilated coronary sinus

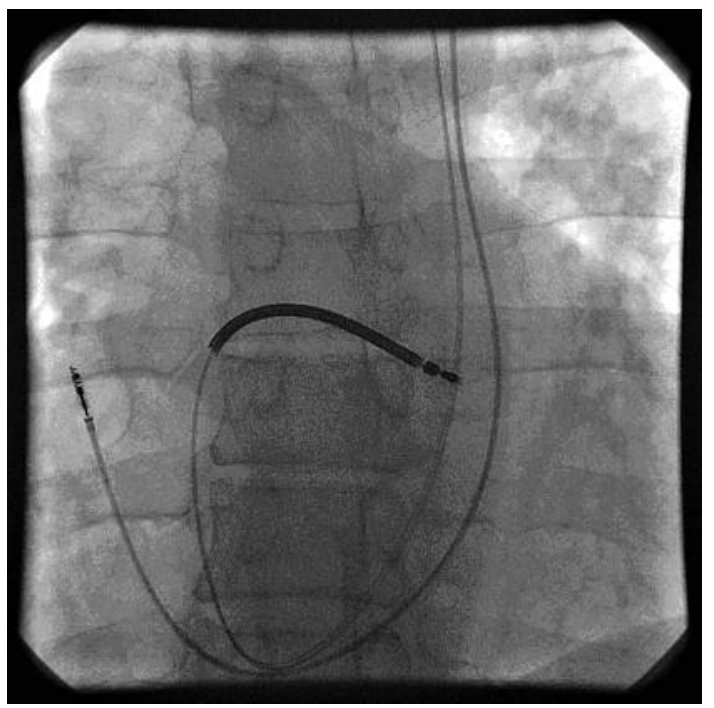

Figure 2. Antero-posterior (AP) view. Screw-in ventricular defibrillator electrode is positioned on the high septum of the right ventricle. Atrial lead is screwed in the high lateral wall of the right atrium
Switzerland) was used and introduced into the right atrium, then a U-shape stylet was formed and through a wide loop within the right atrium the lead was easily introduced and fixed to the high septum of the right ventricle. Measurements confirmed good electrical parameters: R-wave was $14.0 \mathrm{mV}$ and stimulating threshold was $0.6 \mathrm{~V} / 0.5 \mathrm{~ms}$. Through the next puncture of the left subclavian vein and PLSVC the atrial active fixation lead Optisense 1699TC (St. Jude Medical, St. Paul, MN, USA) was introduced and screwed in the high lateral wall of the right atrium. In this position, the electrical parameters were also acceptable: P-wave was $1.6 \mathrm{mV}$, and atrial stimulating threshold was 0.5 V/0.5 ms. Finally a dual chamber ICD Medtronic Maximo DR (Medtronic, Tolochenaz, Switzerland) was implanted in the left pectoral region. The total procedure time was similar to that of the classical ICD implantation - 1 hour and $30 \mathrm{~min}$. Fluoroscopy time was also brief $-5 \mathrm{~min}$. No complications were encountered during the procedure.

Electrical parameters were controlled 24 hours a day for the duration of 7 days after implantation and were comparable to those during the operation. A chest X-ray also confirmed the stable location of leads. The defibrillation threshold test was performed 7 days after implantation. Induced ventricular fibrillation was successfully recognized and terminated by $20 \mathrm{~J}$ shock. Because the right ventricular lead is located in an atypical position (Figures 2, 3) - the coil goes across the tricuspid valve and the lead tip is fixed close to the tricuspid annulus - we recommended frequent follow-up visits. The next follow-up visit is planned for one month after implantation.

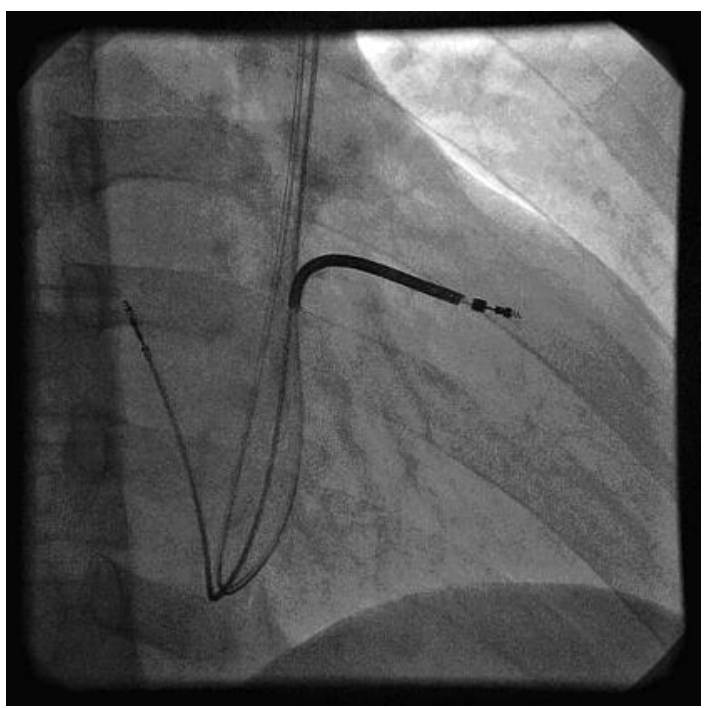

Figure 3. Right ventricular defibrillation lead and atrial lead location. Right anterior oblique (RAO) $30^{\circ}$ view 


\section{Discussion}

There are a lot of publications about successful pacemaker or defibrillator lead implantations via PLSVC [2-8]. The major obstacle for this procedure is right ventricular lead implantation due to the tip of the lead deflecting away from the tricuspid annulus. Approaches to overcome this issue include: manual reshaping of the stylet into a U-shaped stylet, necessitating considerable manoeuvring, forming a loop in the right atrium using the right atrial free wall for support, ventricular lead placement in the left ventricular branch of the coronary sinus and use of an atrial "j" stylet for ventricular lead placement [2]. Konstantino et al. reported a technique of ICD lead implantation via PLSVC using a coronary sinus delivery system as used today in cardiac resynchronization therapy [9]. The majority of authors presented implantation via PLSVC as technically difficult, time consuming and requiring prolonged fluoroscopy. Biffi et al. compared patients implanted through LSVC and classically; the fluoroscopy time was similar to right sided implants $(480 \pm 18$ s vs. $460 \pm 127$ s, $P=\mathrm{NS}$ ) but procedure time was longer (195 \pm 7 vs. $120 \pm 29 \mathrm{~min}, P<0.03)$ [2]. In the manuscript presented by Ratliff et al. the fluoroscopy time varies from 5 to $20 \mathrm{~min}$ and procedure time from 70 to $225 \mathrm{~min}$ [1]. In our case we showed that anatomical conditions of some patients with PLSVC are not always a substantial obstacle for successful implantation considering also the similar X-ray exposure and procedure time as that of the classical approach. Therefore we suggest that implantation via PLSVC should be performed in any case when diagnosis is made intraoperatively unless catheter handling appears difficult or fluoroscopy time exceeds a defined time by the operator. Left-side implantation ICDs may have a lower defibrillation threshold compared to right-sided placement [10]. Current ICD generations are also capable of achieving acceptable defibrillation thresholds when placed in the right pectoral.

We conclude that in the era of increasing procedures with device implantation operators should be aware of the possibility of PLSVC. After the left subclavian vein is punctured the guide wire takes an unusual left-sided downward course, crossing to the coronary sinus or directly to the right atrium. Not every case introduces and manipulates the lead complex. PLSVC in general does not prevent successful placement of an active fixation ICD lead and left-side device implantation. If PLSVC is recognized intra-operatively we suggest continuing the transvenous left-sided approach and considering a right venous access in case of failure to reach a convenient site.

\section{References}

1. Ratliff HL, Yousufuddin M, Lieving WR, et al. Persistent left superior vena cava: case reports and clinical implications. Int J Cardiol 2006; 113: 242-6.

2. Biffi M, Boriani G, Frabetti L, Bronzetti G, Branzi A. Left superior vena cava persistence in patients undergoing pacemaker or cardioverter-defibrillator implantation: a 10year experience. Chest 2001; 120: 139-44.

3. Paperini L, Pardini E, Ebert AG, Galli M. Transvenous cardioverter-defibrillator implantation with a double coil lead via persistent left superior vena cava. Ital Heart J 2004; 5: 711-3.

4. Lappegard KT, Prytz JF, Haug B. Pacemaker implantation in patients with persistent left superior vena cava. Heart Vessels 2004; 19: 153-4.

5. Yasuda M, Nakazato Y, Daida H. ICD lead implantation via persistent left superior vena cava. Heart 2003; 89: 1307.

6. Kempa M, Lubinski A, Wilczek R, Krolak T, Pazdyga A, Raczak G. Cardioverter-defibrillator implantation in a patient with persistent left superior vena cava - a case report. Kardiol Pol 2006; 64: 1281-3.

7. Arora V, Singh J, Kler TS. Implantable cardioverter defibrillatory implantation in a patient with persistent left superior vena cava and right superior vena cava atresia. Indian Heart J 2005; 57: 717-9.

8. Favale S, Bardy GH, Pitzalis MV, Dicandia CD, Traversa M, Rizzon P. Transvenous defibrillator implantation in patients with persistent left superior vena cava and right superior vena cava atresia. Eur Heart J 1995; 16: 704-7.

9. Konstantino Y, Kusniec J, Shohat-Zabarski R, Battler A, Strasberg B. Cardiac defibrillator implantation via persistent left superior vena cava facilitated by a coronary sinus delivery system. Europace 2009; 11: 119-20.

10. Roberts PR, Allen S, Betts T, et al. Increased defibrillation threshold with right-sided active pectoral can. J Interv Card Electrophysiol 2000; 4: 245-9. 